PROCEEDINGS OF THE

AMERICAN MATHEMATICAL SOCIETY

Volume 129, Number 2, Pages 347-350

S 0002-9939(00)05761-0

Article electronically published on August 29, 2000

\title{
ON THE LENGTH OF THE SPECTRAL SEQUENCE OF A LIE ALGEBRA EXTENSION
}

\author{
DONALD W. BARNES
}

(Communicated by Dan M. Barbasch)

\begin{abstract}
The length of the spectral sequence of a Lie algebra extension is at most $1+$ the dimension of the quotient algebra. We show that this bound can be attained for arbitrarily large quotient algebras even when the algebra is nilpotent and the extension splits.
\end{abstract}

\section{INTRODUCTION}

The spectral sequences $\left\{\left(E^{r}, d^{r}\right) \mid r=0,1, \ldots\right\}$ considered in this paper all terminate, that is, there exists $t$ such that $E^{r}=E^{t}$ for all $r \geq t$. This stable value is denoted by $E^{\infty}$. We define the length $l$ of the spectral sequence to be the smallest $t$ for which $E^{t}=E^{\infty}$. Note that $d^{r}=0$ for $r \geq l$, but $d^{l-1} \neq 0$.

Let $L$ be a Lie algebra over the field $F, M$ an ideal of $L$ and let $V$ be an $L$ module. We consider the Hochschild-Serre spectral sequence of this extension $L$ of $M$ by $L / M$, working with homology rather than cohomology to simplify the notation. The terms $\left(E^{0}, d^{0}\right)$ and $\left(E^{1}, d^{1}\right)$ depend on the choices of resolutions used in the construction of the spectral sequence, and

$$
E_{p q}^{2}=H_{p}\left(L / M, H_{q}(M, V)\right) .
$$

Thus for $r \geq 2$, non-zero terms $E_{p q}^{r}$ are confined to the rectangle $0 \leq p \leq \operatorname{dim}(L / M)$ and $0 \leq q \leq \operatorname{dim}(M)$. Since $d_{p q}^{r}$ maps $E_{p q}^{r}$ into $E_{p-r, q+r-1}^{r}$, for it to be non-zero, we must have $r \leq \operatorname{dim}(L / M)$ and $r-1 \leq \operatorname{dim}(M)$. Thus the length $l$ of the spectral sequence always satisfies

$$
l \leq 1+\operatorname{dim}(L / M) \text { and } l \leq 2+\operatorname{dim}(M)
$$

The Hochschild-Serre spectral sequence is discussed at length in Barnes [3], with the particular resolution and filtration used here appearing in section IV.4 of [3]. Like most references, this concentrates on cohomology. The homology version is mentioned briefly in Cartan and Eilenberg [4, p. 351] and in Barnes [1]. The dual $\bar{V}=\operatorname{Hom}_{F}(V, F)$ of a right $L$-module $V$ is a left $L$-module, with the action of $x \in L$ on $f \in \bar{V}$ given by $(x f)(v)=f(v x)$ for all $v \in V$. For a left $L$-module projective resolution $P_{\bullet}$ of $F$,

$$
\operatorname{Hom}_{F}\left(V \otimes_{L} P_{\bullet}, F\right) \simeq \operatorname{Hom}_{L}\left(P_{\bullet}, \operatorname{Hom}_{F}(V, F)\right)=\operatorname{Hom}_{L}\left(P_{\bullet}, \bar{V}\right) .
$$

Received by the editors April 16, 1999.

1991 Mathematics Subject Classification. Primary 18G40, 17B56.

Key words and phrases. Spectral sequence, Lie algebras. 
Thus dualisation converts a complex for the homology of $V$ into a complex for the cohomology of $\bar{V}$ and $\operatorname{Hom}_{F}\left(H_{n}(L, V)\right) \simeq H^{n}(L, \bar{V})$. Any ascending filtration of $V \otimes_{L} P \bullet$ gives a descending filtration of the cochain complex, and the dual of the homology spectral sequence for $V$ is the cohomology spectral sequence for $\bar{V}$. Thus the results of this paper apply also to the cohomology spectral sequence.

\section{SHARPER BOUNDS}

In this section, we impose conditions on the algebra $L$, the ideal $M$ and the module $V$ to obtain sharper bounds on the length $l$. We suppose that $L$ splits over $M$. We have a subalgebra $U$ of $L$ which complements $M$. We choose a basis $\left\{u_{1}, \ldots, u_{n}\right\}$ of $U$ and a basis $\left\{x_{1}, \ldots, x_{m}\right\}$ of $M$. We work with the complex $C_{\bullet}=V \otimes \Lambda(L)$, where

$$
\begin{aligned}
d\left(v \otimes\left\langle\alpha_{1}, \ldots, \alpha_{r}\right\rangle\right)= & \sum_{i=1}^{r}(-1)^{i-1} v \alpha_{i} \otimes\left\langle\alpha_{1}, \ldots \widehat{i} \ldots, \alpha_{r}\right\rangle \\
& +\sum_{i<j}(-1)^{i+j} v \otimes\left\langle\left[\alpha_{i}, \alpha_{j}\right], \alpha_{1}, \ldots \widehat{i} \ldots \widehat{j} \ldots, \alpha_{r}\right\rangle
\end{aligned}
$$

with the filtration $\Phi$ given by

$$
v \otimes\left\langle\alpha_{1}, \ldots, \alpha_{r}\right\rangle \in \Phi^{p} C_{r} \text { if at most } p \text { of the } \alpha_{i} \text { lie outside } M \text {. }
$$

Lemma 1. Suppose $L$ splits over $M$ and that $M$ acts trivially on $V$. Then $d_{p 0}^{r}=0$ for $r \geq 2$, and $l \leq 1+\operatorname{dim}(M)$.

Proof. If $d_{p 0}^{r} \neq 0$, then there exists $c \in \Phi^{p} C_{p}$ with $d c \in \Phi^{p-r} C_{p-1}$ and $d c \notin$ $d \Phi^{p-1} C_{p}+\Phi^{p-r-1} C_{p-1}$. We express $c$ as the sum $c=c^{\prime}+c^{\prime \prime}$ where $c^{\prime}$ is the sum of the terms of the form $v \otimes\left\langle u_{i_{1}}, \ldots, u_{i_{p}}\right\rangle$ and $c^{\prime \prime}$ is the sum of the terms of the form $v \otimes\left\langle u_{i_{1}}, \ldots, u_{i_{p-t}}, x_{j_{1}}, \ldots, x_{j_{t}}\right\rangle$ with $t \geq 1$. For each term of this latter type, every non-zero term in its boundary involves at least one of the $x_{j}$. It follows that $d c^{\prime \prime} \in \Phi^{p-2} C_{p-1}$, so also $d c^{\prime} \in \Phi^{p-2} C_{p-1}$. But $d c^{\prime}$ consists of terms which do not involve any of the $x_{j}$, so $d c^{\prime}=0$ and $d c=d c^{\prime \prime} \in d \Phi^{p-1} C_{p}$ contrary to assumption. Since a non-zero arrow cannot start on the lower edge of the rectangle, we have $l \leq 1+\operatorname{dim}(M)$.

Theorem 2. Suppose $L$ splits over $M$, and that $M$ is nilpotent and acts trivially on $V$. Then the spectral sequence has length at most the greater of 2 and $\operatorname{dim}(M)$.

Proof. By Lemma 1, we have that, for $r \geq 2$, a non-zero arrow $d_{p q}^{r}$ cannot start on the lower edge of the rectangle. We show that, under our additional assumption that $M$ is nilpotent, it also cannot end on the upper edge of the rectangle. Suppose $d_{p q}^{r} \neq 0, r \geq 2$ and that $d_{p q}^{r}$ ends on the upper edge of the rectangle. Then $q=m-r+1$. There exists $c \in \Phi^{p} C_{p+q}$ such that $d c \in \Phi^{p-r} C_{p+q-1}$ and $d c \notin$ $d \Phi^{p-1} C_{p+q}+\Phi^{p-r-1} C_{p+q-1}$. Now $c=c^{\prime}+c^{\prime \prime}$ where $c^{\prime}$ is a sum of terms of the form $v \otimes\left\langle u_{i_{1}}, \ldots, u_{i_{s}}, x_{j_{1}}, \ldots, x_{j_{m-t}}\right\rangle$ where $s=p+q-m+t$ and $t \geq 1$, and $c^{\prime \prime}$ is a sum of terms of the form $v \otimes\left\langle u_{i_{1}}, \ldots, u_{i_{p-r+1}}, x_{1}, \ldots, x_{m}\right\rangle$. Since $M$ is nilpotent, the $x_{j}$ may be chosen such that for any $\alpha \in M,\left[\alpha, x_{j}\right]$ is in the space spanned by $x_{j+1}, \ldots, x_{m}$. It follows that the only non-zero terms in $d v \otimes\left\langle u_{i_{1}}, \ldots, u_{i_{p-r+1}}, x_{1}, \ldots, x_{m}\right\rangle$ involve all $m$ of the $x_{j}$. But $d c^{\prime}$ consists of terms involving fewer than $m$ of the $x_{j}$, while $d c$ by assumption, consists only of terms involving all the $x_{j}$. Thus $d c^{\prime}=0$, and $d c=d c^{\prime \prime} \in d \Phi^{p-1} C_{p+q}$ contrary to assumption. 
If $M$ is nilpotent, any $L$-module $V$ is the direct sum $V_{0} \oplus V_{1}$ of $L$-submodules with $M$ acting nilpotently on $V_{0}$ and with $V_{1}^{M}=0$. As $H_{q}\left(M, V_{1}\right)=0$ for all $q$, (see Dixmier [5, Theorème 1], see also Barnes [1, Theorem 1] and [2]), $E_{p q}^{2}\left(V_{1}\right)=0$ for all $p, q$, and $E^{2}\left(V_{1}\right)=E^{\infty}\left(V_{1}\right)$ trivially. Only the null component $V_{0}$ can give a spectral sequence of length greater than 2. Our next result gives a bound which does not depend on the dimensions of $M$ or $L / M$.

Theorem 3. Suppose $L$ splits over $M$, that $M$ is abelian and acts trivially on $V$. Then the spectral sequence has length at most 2.

Proof. Every non-zero term in $d v \otimes\left\langle u_{i_{1}}, \ldots, u_{i_{p}}, x_{j_{1}}, \ldots, x_{j_{q}}\right\rangle$ involves exactly $q$ of the $x_{j}$. Thus $C \bullet$ is in fact graded by $\Phi$, with $v \otimes\left\langle u_{i_{1}}, \ldots, u_{i_{p}}, x_{j_{1}}, \ldots, x_{j_{q}}\right\rangle$ a homogeneous element of degree $p$, and $d$ reducing grade by 1 . It follows that $d^{1}$ is the only possibly non-zero differential in the spectral sequence.

\section{EXAMPLES}

In both the following examples, $L$ is nilpotent of class 2 , splits over $M$ and acts on the 1-dimensional trivial module. The trick used to make the calculation of the length reasonably simple is to arrange that each $d\left\langle\alpha_{1}, \ldots, \alpha_{t}\right\rangle$ which must be calculated has at most two non-zero terms. The first example, unfortunately of low dimension, achieves both the bounds $l=1+\operatorname{dim}(L / M)$ and $l=\operatorname{dim}(M)$.

Example 1. Let $U$ and $M$ have bases $\{u, v\}$ and $\{x, y, z\}$ respectively, with the multiplication given by $[u, x]=[v, y]=[x, y]=z$ and all other products 0 . Then the spectral sequence has length $l=3$.

Proof. We have $d(\langle u, v, x\rangle+\langle v, x, y\rangle)=\langle z, x\rangle \in \Phi^{0} C_{2}$. Since for any $\alpha, \beta \in L$, $d\langle\alpha, \beta, z\rangle=0, d \Phi^{1} C_{3}$ is spanned by $d\langle u, x, y\rangle=\langle y, z\rangle+\langle u, z\rangle$ and $d\langle v, x, y\rangle=$ $\langle v, z\rangle-\langle x, z\rangle$, so $\langle z, x\rangle \notin d \Phi^{1} C_{3}$. Thus $d_{21}^{2}: E_{21}^{2} \rightarrow E_{02}^{2}$ is non-zero and $l=3=$ $1+\operatorname{dim}(L / M)=\operatorname{dim}(M)$.

Example 2. Let $M$ have basis $\left\{v_{1}, \ldots, v_{n}, w_{1}, \ldots, w_{n-1}, z_{1}, \ldots, z_{n}\right\}$ and let $U$ have basis $\left\{u_{1}, \ldots, u_{n}\right\}$, with the multiplication given by $\left[u_{i}, v_{i}\right]=z_{i}=\left[w_{i}, v_{i+1}\right]$ and all other products 0 . Then the spectral sequence has length $l=n+1$.

Proof. We put $\alpha_{0}=0$,

$$
\alpha_{i}=\left\langle z_{i}, w_{1}, \ldots, w_{i-1}, u_{i+1}, \ldots, u_{n}\right\rangle \text { and } c_{i}=\left\langle w_{1}, \ldots, w_{i-1}, v_{i}, u_{i}, \ldots, u_{n}\right\rangle .
$$

Then $d c_{i}=-\alpha_{i-1}+\alpha_{i}$. Observe that $c_{1}+\ldots+c_{n} \in \Phi^{n} C_{n+1}$ and that

$$
d\left(c_{1}+\ldots+c_{n}\right)=\alpha_{n} \in \Phi^{0} C_{n}
$$

We show that $\alpha_{n} \notin d \Phi^{n-1} C_{n+1}$.

$C_{n}$ has a basis consisting of the $n$-fold wedge products of members of our basis of $L$. Let $A$ be the subspace of $C_{n}$ spanned by the $\alpha_{i}$, and let $B$ be the subspace spanned by the other $n$-fold wedge products. Let $\pi: C_{n} \rightarrow A$ be the projection onto $A$ defined by the direct decomposition $C_{n}=A \oplus B$. We similarly decompose $C_{n+1}$ into the direct sum $C \oplus D$ where $C$ is spanned by the $c_{i}$ and $D$ by the other $(n+1)$-fold wedge products. Of the factors in $\alpha_{i}$, only $z_{i}$ is expressible as a product of basis elements, and that, only in two ways. Thus $c_{i}$ and $c_{i+1}$ are the only $(n+1)$-fold wedge products of basis elements whose boundaries have $\alpha_{i}$ as a term. If $\gamma \in D$, then $d \gamma \in B$ and $\pi d \gamma=0$. 
If $c \in \Phi^{n-1} C_{n+1}$, then $c=\sum_{i=2}^{n} \lambda_{i} c_{i}+\gamma$ where $\gamma \in D$ and $\lambda_{i}$ are elements of the field. We then have

$$
d c=\sum_{i=2}^{n} \lambda_{i}\left(-\alpha_{i-1}+\alpha_{i}\right)+d \gamma
$$

and

$$
\pi d c=\sum_{i=2}^{n} \lambda_{i}\left(-\alpha_{i-1}+\alpha_{i}\right) \neq \alpha_{n}
$$

Thus $d c \neq \alpha_{n}$ and $\alpha_{n} \notin d \Phi^{n-1} C_{n+1}$. Hence $d_{n 1}^{n}: E_{n 1}^{n} \rightarrow E_{0 n}^{n}$ is non-zero and $l=n+1$.

\section{Questions}

Several interesting questions remain unanswered. In Theorem 2 , is $\operatorname{dim}(M)$ the best possible bound? Example 2 only achieved $l=2+$ integral part of $\operatorname{dim}(M) / 3$. What happens in Lemma 1 and Theorems 2 and 3 if we weaken the condition that $M$ acts trivially to $M$ acts nilpotently? If $V$ has a submodule $W$, is $l(V)$ bounded by some function of $l(W)$ and $L(V / W)$ ?

\section{REFERENCES}

[1] D. W. Barnes, On the cohomology of soluble Lie algebras, Math. Zeitschr. 101 (1967), 343349. MR 36:3836

[2] Sortability of representations of Lie algebras, J. Alg. 27 (1973), 486-490. MR 51:3242

[3] Spectral sequence constructors in algebra and topology, Mem. Amer. Math. Soc. 53 No. 317, 1985. MR 86e:55032

[4] H. Cartan and S. Eilenberg, Homological algebra, Princeton University Press, 1956. MR 17:1040e

[5] J. Dixmier, Cohomologie des algèbres de Lie nilpotentes, Acta Sci. Math. Szeged. 16 (1955), 246-250. MR 17:645b

1 Little Wonga Road, Cremorne, New South Wales 2090, Australia

E-mail address: donb@netspace.net.au 